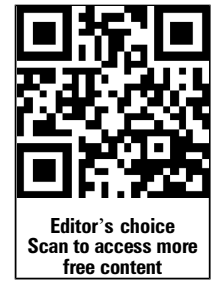

Renal Section, Department of Medicine, Boston University School of Medicine, Boston, Massachusetts, USA

Correspondence to Dr Craig E Gordon, Renal Section, Boston University Medical Center, Boston, MA 02118, USA; craig. gordon@bmc.org

Received 4 April 2013 Revised 17 January 2014 Accepted 14 February 2014

Published Online First 5 March 2014

CrossMark

To cite: Gordon $\mathrm{CE}$, Borkan SC. Postgrad Med J 2014:90:267-272.

\title{
Recapturing time: a practical approach to time management for physicians
}

\author{
Craig E Gordon, Steven C Borkan
}

\section{ABSTRACT}

Increasing pressures on physicians demand effective time management and jeopardise professional satisfaction. Effective time management potentially increases productivity, promotes advancement, limits burnout and improves both professional and personal satisfaction. However, strategies for improving time management are lacking in the current medical literature. Adapting time management techniques from the medical and nonmedical literature may improve physician time management habits. These techniques can be divided into four categories: (1) setting short and long-term goals; (2) setting priorities among competing responsibilities; (3) planning and organising activities; and (4) minimising 'time wasters'. Efforts to improve time management can increase physician productivity and enhance career satisfaction.

\section{INTRODUCTION}

Maintaining work-life balance as a physician necessitates learning how to improve efficiency and effectively manage the limited resource of available time. Indeed, pressures on physicians have recently increased, partly due to changes in physician reimbursement and a decrease in the availability of funding to support scholarly and patient-centred endeavours. Physicians currently manage a growing number of increasingly complex patients in less time, deal with a larger volume of administrative paperwork, and are more easily accessible via email, pagers, mobile phones and other technological advances, all factors contributing to high rates of professional burnout. ${ }^{1-6}$ As a result, physicians increasingly report major challenges in managing time demands. ${ }^{1} 378$ However, few medical publications directly address the problem of how to improve time management (TM) skills for physicians. ${ }^{7-11}$ Although business, industry and the legal professions acknowledge the importance of TM training, there are few current TM training seminars available to physicians.

Although some highly successful physicians appear to have limitless time, it is actually the management rather that the absolute amount of time that varies. Simply stated, physicians must identify effective TM approaches to promote their professional success and personal satisfaction. Without these, all aspects of one's career are adversely affected, including clinical productivity, success as an administrator or teacher, and meeting important deadlines. Lack of success in these areas impedes growth within an institution or practice, slows promotion and undermines recognition, ultimately diminishing physician job satisfaction. The resulting stress impacts the physician's personal life, further undermining the overall sense of satisfaction and increasing the risk of burnout. ${ }^{12}$ It is well recognised that physicians experience high rates of depression and suicide, as well as burnout, ${ }^{4}{ }^{13}$ perhaps partly related to poor TM practices. On a broader scale, academic medical centres, national professional organisations and the public should pay attention because each of these entities benefit greatly from effective and successful physicians and therefore might be expected to assist physicians in developing effective TM skills. Paradoxically, there are few published studies in the medical literature to assist physicians in improving TM techniques.

To identify TM techniques for this review, we performed a Medline search using the terms 'time management', 'physician', 'burnout' and 'career development'. With this approach (figure 1), 5624 studies were identified and reviewed by title and abstract. Of these, 5412 studies were excluded as unrelated to TM for physicians. Although most of the remaining 212 studies alluded to the importance of developing effective TM skills to manage stress or burnout (45) and in early career faculty development (41), and suggested that effective TM is a competency for physician excellence (26), few published studies provided specific recommendations for implementing effective TM skills. Indeed, only 15 publications, books or electronic references described TM techniques for physicians. These publications provided the evidence base for this review.

We combine resources from the medical and non-medical literature to identify TM techniques appropriate for the medical profession. If implemented, these principles are likely to improve physicians' management of professional demands, increase career satisfaction and improve work-life balance. Effective TM strategies can be separated into four distinct principles: (1) setting short and long-term goals; (2) setting priorities among competing responsibilities; (3) planning and organising activities; and (4) minimising 'time wasters'. We propose specific strategies for physicians to implement for each category. A discussion of the important topic of effective TM techniques in the clinical arena is beyond the scope of this review.

\section{SETTING SHORT AND LONG-TERM GOALS}

One common practice of highly effective professionals is to frequently set and revise goals. These goals can be divided into short and long-term aims. Experts define short-term goals as achievable within 1-3 years, whereas long-term goals typically require $5-10$ years. ${ }^{14}$ One common physician error is to allow long-term goals to be dominated by more immediate and urgent short-term time 


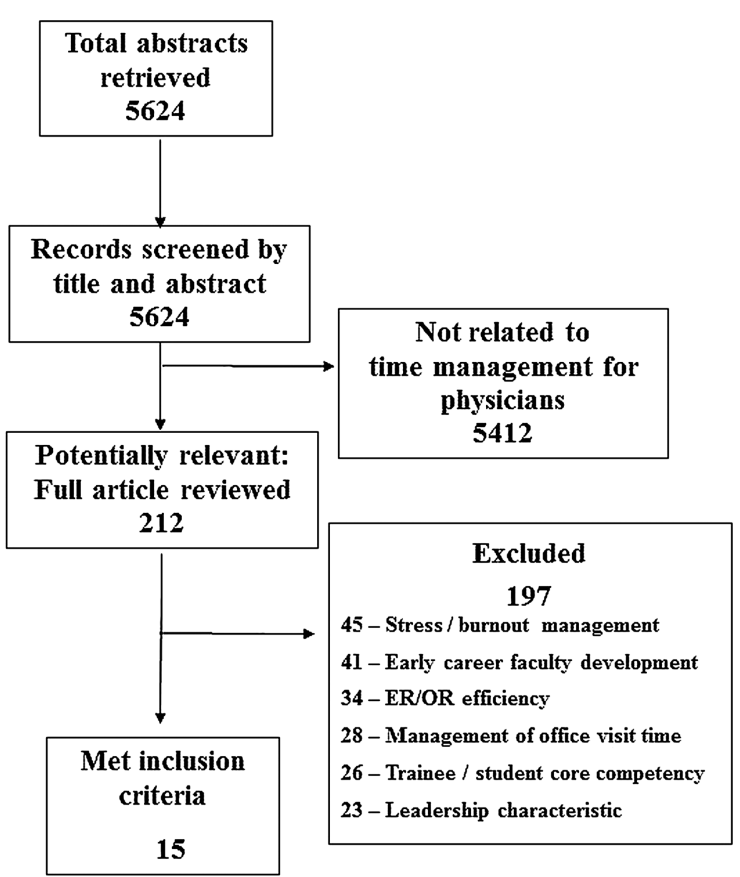

\begin{tabular}{|l|l|}
\hline TM principle & References \\
\hline 1. Goal setting & 14 \\
\hline 2. Setting priorities & $11,15,16,18,20,21,22$ \\
\hline 3. Planning/organizing & $7,9,10,14,16,20,21,23,25,26$ \\
\hline 4. Minimizing time wasters & $7,9,10,14,15,16,20,22,23,27$ \\
\hline
\end{tabular}

Figure 1 Literature search and studies contributing to review. ER, emergency room; OR, operating room; TM, time management.

demands. Regular review of long-term goals promotes thoughtful decision-making as new short-term opportunities appear. Identifying and protecting time required to set and accomplish long-term goals facilitates enduring professional success.

Admittedly, achieving long-term goals might require completing a series of short-term milestones. For example, young physician-scientists are required to devise studies, publish manuscripts, present at national meetings, and submit grant applications as interim steps towards academic promotion. A different set of short-term goals is required for colleagues aspiring to educational leadership positions or clinical directorships. Significant experience and maturity are required to balance and revise these short-term goals en route to long-term achievement. Therefore, most physicians benefit from periodic review by a trusted mentor and/or colleague to assess progress on short-term accomplishments on the path towards achieving long-term goals.

\section{Principles of goal setting}

Honest reality testing is required to determine whether goals are achievable based upon the physician's skills and current professional standing. This exercise is important since the pursuit of unrealistic or conflicting goals ultimately leads to frustration and professional dissatisfaction. Alternatively, setting goals too conservatively promotes stagnation and ineffective use of professional talent and may also limit professional satisfaction.

Professional growth and promotion may appear overwhelming or unachievable without consideration of their component parts. How many lectures, publications, teaching or patient encounters are required for advancement? Selecting intermediary achievements on the path to long-term goals facilitates realistic determination of their ultimate achievability. A young physician's career may stall without a career plan that clearly defines intermediate goals required for advancement. In part, the target largely defines the intermediary steps required to achieve it. For example, if departmental chairperson is the ultimate goal, then becoming an independent investigator with national or international recognition, heading key hospital committees, and establishing educational leadership are appropriate short-term goals. If the goal is clinical director of the practice, a different set of goals is necessary and might include serving as quality improvement director, chair of a clinical access committee or director of population management. The importance of identifying and monitoring short-term goals increases as aspirations become more ambitious.

The discipline to set long-term goals is exemplified by the novice runner training for a marathon. Each 1, 2 or 5 -mile run is a crucial and measurable step toward the long-term goal of running the full marathon distance. In an analogous fashion, a successful physician monitors short-term accomplishments on the path towards achieving long-term goals. Periodic and repeated reality testing provides the opportunity for internal and external feedback to assess whether the physician is on track to achieving long-term goals. Specifically, is the pace of achieving interim goals on schedule and is the long-term goal realistic? Do external demands, both professional and personal, necessitate goal revision?

\section{SETTING PRIORITIES AMONG COMPETING RESPONSIBILITIES \\ New responsibilities}

The daily routine of physicians includes managing numerous existing responsibilities and expectations as well as requests for new opportunities and additional responsibilities. Successful TM requires separating 'the wheat from the chaff' among these opportunities. Requests for physician's time are often made without knowledge of pre-existing time demands and assume that every activity is of equal importance and value. Awareness of the physician's short and long-term goals determines which activities are most likely to achieve progress. Before agreeing to new requests, physicians must determine whether the new committee service, lecture, clinical responsibility or research application will advance or retard progress towards short and long-term goals. The answer to this question will determine whether time should be devoted to the new activity or if a polite but firm 'No, but thank you for this opportunity' is the most appropriate reply. ${ }^{11} 1516$

However, a polite decline is not always appropriate when a superior issues the request. In this case, before agreeing to the request, the physician should ask 'What must I give up in order to accept this new responsibility?' Alternatively, it may be possible to accept the new responsibility for a limited period of time or delay its acceptance until other key goals have been achieved. Each of these responses opens a dialogue for negotiation as an alternative to reflexive acceptance of responsibilities that may be irrelevant or even antagonistic to the physician's central goals. Successful balancing of changing responsibilities and goals promotes growth and professional satisfaction.

\section{Existing responsibilities}

Balancing multiple existing responsibilities is a common physician challenge. Covey's Time Management Matrix Technique 
Table 1 Covey's Time Management Matrix Technique (TMMT) for physicians

\begin{tabular}{|c|c|c|}
\hline & Important & Less important* \\
\hline Urgent & $\begin{array}{l}\text { - Page concerning patient with chest pain } \\
\text { Grant submission due in } 48 \mathrm{~h} \\
\text { - National meeting presentation due in } 3 \text { days } \\
\text { Upcoming annual meeting with chief to review goals and responsibilities }\end{array}$ & $\begin{array}{l}\text { III } \\
\text { Routine patient phone calls } \\
\text { Immediate patient billing } \\
\text { Routine medication refills } \\
\text { Complete trainee evaluation forms } \\
\text { Interview medical school candidates }\end{array}$ \\
\hline Less urgent* & $\begin{array}{l}\text { II Grant submission due in 3-6 months } \\
\text { - New innovative educational curriculum } \\
\text { Write review article } \\
\text { Document patient encounters in EMR } \\
\text { - Committee chairperson to prepare agenda for upcoming meeting } \\
\text { - Planning new clinical quality improvement initiative } \\
\text { Review long-term career goals }\end{array}$ & $\begin{array}{l}\text { IV } \\
\text { Email } \\
\text { Non-urgent letters of recommendation } \\
\text { Routine paperwork }\end{array}$ \\
\hline
\end{tabular}

(TMMT) provides a useful construct for prioritising existing responsibilities. ${ }^{14}$ 17-19 The TMMT asks the professional to classify each responsibility as 'Important' or 'Non-important' and 'Urgent' or 'Non-urgent'. ${ }^{17}$ This determination guides the construction of a $2 \times 2$ table (table 1 ). Although physician tasks may appear at first to be equally 'important', effective TM requires that tasks be prioritised by their relative importance for achieving career goals. ${ }^{14-16}$

In medicine, 'urgent' patient care issues often interfere with effective TM by displacing work on 'important' projects that are central to career development. This conflict has given rise to the concept of 'protected time' in which non-clinical goals are less readily interrupted by clinical necessity. However, even physicians with limited protected time can successfully employ the principles of Covey's TMMT to prioritise tasks in relation to their individual goals.

To acknowledge the challenge that physicians face in labelling any of their tasks as 'non-important' or 'non-urgent', we slightly adapted Covey's TMMT by referring to these tasks as 'less important' or 'less urgent'. Thus, the four quadrants in our revised TMMT are: I-Important and urgent; II-Important and less urgent; III-Less important and urgent; and IV-Less important and less urgent (table 1). Accurate and honest placement of responsibilities into one of the four quadrants encourages realistic comparisons of daily, weekly or monthly tasks. This act itself focuses attention and time allocation on activities in quadrants I and $\mathrm{II}^{17}$ the key tasks that promote advancement towards long-term achievement. In particular, efforts devoted to 'important but less urgent' (quadrant II) activities represent the most efficient use of time and energy but often necessitate adequate blocks of time to permit concentration sufficient for the required creativity, planning and implementation. Admittedly, the physician's priorities may be forced into quadrant I as unexpected clinical demands or urgent deadlines arise. However, the imminent deadlines and long hours associated with these quadrant I ('important and urgent') activities are most often accompanied by stress and exhaustion that negatively impact the completion of other tasks. Left unchecked, exclusive attention to short-term, quadrant I and IV demands leads to the perception of perpetual challenge without the satisfaction of measurable progress toward long-term goals. ${ }^{18}$
Productive physicians increase the amount of time devoted to quadrant II activities, which are longer-term projects with distant deadlines. However these activities are the key to achieving long-term goals and aspirations. ${ }^{19}{ }^{20}$ Because these tasks are 'non-urgent', the very activities most likely to promote achievement are frequently avoided until they become 'urgent'. Notably, the primary threats to quadrant II activities come from the two 'urgent' quadrants (quadrants I and III). While urgent and important activities frequently acquire the highest priority, successful TM depends upon progress on important but less urgent ones (quadrant II) in lieu of less important but urgent activities (quadrant III). ${ }^{21}$ For example, scheduling time each week for writing manuscripts, preparing grant proposals, developing a new educational curriculum or for administrative efforts to improve clinical practice before committing time to other urgent but less important time demands is an effective TM technique. ${ }^{1022}$

Admittedly, physician activities categorised as 'less important' or 'less urgent' may be perceived as 'important' and 'urgent' to others, including colleagues and superiors. While ignoring these activities is not advisable, some of these requests can be negotiated to a lower priority. Several suggestions to recapture 'lost time' are provided below, as a mechanism to find time to complete 'less important' tasks. Another alternative is to delegate tasks to others. ${ }^{2022}$ Guilt-free delegation requires the realisation that the task being delegated in many cases provides experience and career growth to the recipient. ${ }^{16}$

\section{PLANNING AND ORGANISING ACTIVITIES Finding time for important activities}

After goals are selected and activities categorised by their relative importance and urgency, the next step is to determine how to most effectively complete key tasks. This requires advanced planning that extends beyond a daily calendar which does not allow adequate preparation and organisation for achieving short or longterm goals. 'One day only' planning readily becomes reactionary, inadvertently shunting attention towards 'urgent' activities. Developing a weekly or monthly schedule is a more effective strategy for achieving long-term goals. ${ }^{23}$ The advantage of this proactive approach is that it identifies available time in advance that can be devoted to 'important' but 'less urgent' activities. 
Table 2 Differentiating tasks by size with descriptions

\begin{tabular}{|c|c|c|}
\hline Size of task (estimated time) & Description & Examples \\
\hline Large $(>1 \mathrm{~h})$ & $\begin{array}{l}\text { Require maximal concentration and uninterrupted time } \\
\text { - Schedule task during most alert and productive time }\end{array}$ & $\begin{array}{l}\text { - Grant application or manuscript writing } \\
\text { - Performing bench or clinical research } \\
\text { Curriculum development } \\
\text { - Proposal for new clinical activity }\end{array}$ \\
\hline Medium (30-60 min) & $\begin{array}{l}\text { Require concentration } \\
\text { - Ideally, should be alert and productive }\end{array}$ & $\begin{array}{l}\text { - Clinical documentation (EMR) } \\
\text { - Reviewing and editing section of manuscript } \\
\text { - Preparing slides for presentation }\end{array}$ \\
\hline Small (5-10 min) & $\begin{array}{l}\text { Require minimal or brief concentration } \\
\text { Schedule during less alert portions of the day or week } \\
\text { - Use as a transition between large or medium-sized tasks (create a 'mental break') }\end{array}$ & $\begin{array}{l}\text { Returning non-urgent phone calls } \\
\text { Signing discharge summaries } \\
\text { Editing letters of recommendation }\end{array}$ \\
\hline Very small (<5 min) & $\begin{array}{l}\text { Require little concentration } \\
\text { - Useful as 'fill' time while waiting for meeting to begin or on hold on the telephone }\end{array}$ & $\begin{array}{l}\text { Responding to electronic messages } \\
\text { Opening and sorting paper mail } \\
\text { Routine paperwork } \\
\text { - Clinical billing }\end{array}$ \\
\hline
\end{tabular}

Self-reflection permits physicians to identify optimal times for greatest productivity. Attention to individual lifetime learning patterns provides valuable guidance for organising the day, week and month.' 1416 For example, being an 'early morning riser', 'afternoon peaker' or 'night owl' is a distinct character trait that when recognised promotes effectiveness by allowing highly productive times of the day to be consciously linked with the most important projects. ${ }^{1622}$ Some physicians may be most effective early in the week before competing urgent demands appear, whereas others need to 'clear the desk' of responsibilities each week before focusing on quadrant II activities. One key to successful scheduling is to identify and protect the most productive times for focusing on the important and often most challenging tasks.

\section{What period of time is necessary?}

Ensuring that an adequate amount of uninterrupted time is available to complete a measurable component of the task is crucial to success (table 2). Limiting scheduled or unscheduled interruptions is important since initiating or resuming projects often requires significant 'energy of activation' in order to focus attention. For example, writing part of a complicated manuscript, grant or clinical proposal should be reserved for times of highest energy, greatest creativity and, when available, sufficient uninterrupted time to complete the task. ${ }^{10}$ However, a prolonged time period is not necessarily required and waiting for long stretches of free time to appear on the calendar can result in delays in progress. In fact, one effective TM technique to reduce procrastination is to devote a short amount of time (even as little as $30 \mathrm{~min}$ ) every day to a project, thereby creating measureable increments in progress. ${ }^{23-25}$ In contrast, responding to email and telephone calls may be relegated to lower energy times of the day or to brief periods of time between scheduled activities. Planning a week or longer in advance allows one to identify and protect long periods of time for the most important activities. $^{14}$

\section{Selecting the optimal venue for important tasks}

Where can work be accomplished most effectively on projects requiring the greatest concentration? An accurate answer to this question promotes productivity. The optimal venue may be at work, a home office, coffee shop, back porch or elsewhere that affords minimal interruption and distraction and should factor in the physician's period of maximal alertness. ${ }^{25} 26$ Successful physicians overcome peer pressure that would otherwise determine where one 'should' do work; the primary goal is to be productive, not to appease colleagues. The most important tasks deserve a recurring reservation at this optimal location.

\section{How to plan and organise activities}

Effective TM clearly requires organisational skill. Each day, week or month can be planned in advance. Although personal scheduling preferences differ, organising and adhering to a schedule, as well as control and awareness of the calendar, are keys to effective TM. ${ }^{14}$ If Thursdays are completely filled with a busy clinic, multiple meetings or regularly scheduled teaching commitments, then a different day is a far superior choice for manuscript writing or developing new quality improvement initiatives. Organising the calendar by grouping similar activities such as meetings, clinical work or teaching activities in a manner that reserves other larger blocks of time for activities that require more concentration promotes effective $\mathrm{TM}^{7}$ Ideally, effective TM includes blocks of 'reserved time' at least once each week for quadrant II activities. ${ }^{10} 14$

Awareness of the next day's schedule is an effective TM technique. The concept of 'activation energy' to begin a task also applies to each work day. Preparing for the day during the morning's commute or the evening before ensures that valuable workday time is not lost simply due a lack of awareness of planned activities. ${ }^{7}{ }^{20}$ This is especially important before each work week begins in order to identify the available time periods to advance quadrant II activities. ${ }^{20}$ Preparation facilitates progress on daily tasks by immediately attending to them upon arrival at work.

\section{MINIMISING TIME WASTERS}

TM experts have identified numerous potential 'time wasters'. ${ }^{14} 1622$ This term refers to interruptions, distractions and other delays that insidiously reduce productive time. Ten common time wasters relevant for physicians are detailed in table 3 .

\section{Physical interruptions}

A major source of lost time is interruption from colleagues. ${ }^{7}$ These unexpected, and often unintentionally poorly timed, 
Table 3 Ten common time wasters and potential solutions

\begin{tabular}{|c|c|}
\hline Time waster & Proposed solution \\
\hline Telephone calls & Check messages and return calls 1-2 times per day* \\
\hline Email & $\begin{array}{l}\text { Check no more than 3-4 times per day* } \\
\text { Disable auto-alert messages for mail arrivals } \\
\text { Develop a reliable and reproducible filing system for saved } \\
\text { messages } \\
\text { Rapidly dispose of unwanted messages at first pass } \\
\text { Accurately identify and discard junk mail }\end{array}$ \\
\hline $\begin{array}{l}\text { Physical } \\
\text { interruptions }\end{array}$ & Close office door and respect as colleagues do the same \\
\hline Paper & $\begin{array}{l}\text { Handle each piece of paper only once ('When in doubt, } \\
\text { throw it out') } \\
\text { Develop reliable and reproducible filing system } \\
\text { Store publications as electronic PDF files }\end{array}$ \\
\hline Repetitive activities & $\begin{array}{l}\text { Automate (eg, develop patient education handouts) } \\
\text { Create 'quick text' for frequently used phrases in email or } \\
\text { in electronic medical records } \\
\text { Delegate tasks that can be done by others }\end{array}$ \\
\hline Disorganisation & $\begin{array}{l}\text { Clean and organise the desk and office } \\
\text { Organise paper, mail and electronic files for easier } \\
\text { accessibility }\end{array}$ \\
\hline Procrastination & $\begin{array}{l}\text { Identify and address reasons for procrastination } \\
\text { Accomplish small increments of progress on a project } \\
\text { Do not allow perfectionism to get in the way of progress }\end{array}$ \\
\hline Meetings & $\begin{array}{l}\text { Arrive on time (change the culture of lack of punctuality) } \\
\text { Bring alternative work if others are not punctual }\end{array}$ \\
\hline Waitingt & $\begin{array}{l}\text { Perform quick and easy small tasks (clinical or } \\
\text { administrative paperwork works well) }\end{array}$ \\
\hline Commuting & $\begin{array}{l}\text { Enjoy music, books on tape, quiet self-reflection, relax } \\
\text { Use audio continuing medical education, learn a foreign } \\
\text { language } \\
\text { Read journal articles if using public transportation }\end{array}$ \\
\hline
\end{tabular}

visits are particularly problematic not only because they interrupt progress but because the visitor also may request assistance with 'urgent' but 'less important' tasks (quadrant IV) in the physician's TMMT. One effective solution is to close the office door during tasks that require maximal concentration and to open it when contact and collaboration with others is not only welcome but may be mutually beneficial. ${ }^{15}$ Most colleagues learn to respect the message implicit in these actions, allowing the physician to recapture otherwise lost time. Similar respect for colleagues' closed doors minimises their distraction and creates a culture of mutual TM respect. ${ }^{23}$

\section{Technological interruptions}

Email, telephone calls and pagers interrupt physicians in a manner similar to unexpected visitors, ${ }^{10}{ }^{14}$ and are frequent sources of physician complaints. ${ }^{15}$ Improper use of email, ironically designed to save time, is one of the most common physician time wasters. Used correctly, email can be an incredibly powerful TM tool; however, many find it impedes productivity. For example, checking for and responding to new messages every few minutes promotes behaviours that undermine productivity. ${ }^{23}$ Ideally, email should be accessed three to four times per day with 'offline time' scheduled in-between. ${ }^{10}$ Disabling the audible tone that accompanies each new message also avoids distraction and recaptures time. ${ }^{1023}$ Brief, interim periods of time between longer activities are optimal for responding to new messages. ${ }^{23}$ One exception is a job description that necessitates time-sensitive clinical or administrative responses, but in these cases the decision is a conscious one.

Built-in email features allow messages to be organised or automatically deleted and are also ideal for pre-formatted text phrases or signatures that are repeatedly used. ${ }^{23}$ Ideally, electronic messages should be handled only once, a recommendation that also applies to paperwork that crosses the physicians desk. ${ }^{7} 92023$ Unnecessary messages can be discarded immediately, key information can be filed for later review, and only the remainder requires action. ${ }^{23}$ The imperative to handle each message once increases the importance of checking email only a few times per day. Otherwise, the physician loses valuable time by entering a cycle of immediate messaging and re-messaging. ${ }^{10}$

Telephones and pagers are common sources of lost time, particularly during activities that require concentration. Although urgent and important telephone calls or pages must be answered immediately, the majority are less urgent. Call forwarding or screening by a trusted administrator are effective TM techniques. Caller identification and alphanumeric paging also permit selective call monitoring and promote uninterrupted work time, allowing important tasks to be completed.

Proper use of electronic medical record (EMR) systems also enhances TM. ${ }^{15}$ These systems often have automatic 'quick text' features that efficiently document medical information. Typically, these EMR features require significant training time, but when used properly, yield long-lasting efficiency dividends. Voice recognition software, considerably improved in recent years, can also enhance productivity. Other new technologies such as cloud backup systems for important files and articles, synchronised calendars and task lists can improve TM. However, new technology is a double-edged sword; TM may be improved, but if used poorly, TM is compromised.

\section{Hidden time: waiting to be reclaimed}

Regardless of the specific responsibilities, all physicians have 'hidden time', lost while waiting for meetings, conferences or patients. By carrying paperwork, a personal digital assistant, day planner or other tasks that can be completed while waiting, hidden time can be recaptured. ${ }^{15}$ Commuting is another important source of reclaimable time. ${ }^{27}$ Time can be recaptured simply by adjusting the mode of transportation. Physicians on public transportation can read medical journals, edit manuscripts, or review slides for upcoming presentations. Commuting drivers can listen to continuing medical education recordings, learn a new language to expand patient care skills, or reflect on longterm career goals. ${ }^{15}$ Paradoxically, focus on non-career interests or mental relaxation often promotes innovative thinking on current and future projects.

\section{CONCLUSION}

Physicians seeking to improve productivity can identify and practice TM skills that increase efficiency and satisfaction as well as reduce professional and personal stress. Although lifelong behavioural change and vigilance are required to implement and maintain effective TM, readers are encouraged to first identify one or two areas of TM inefficiency and endeavour to improve in these areas. Once these have been successfully addressed, additional TM techniques can be implemented. By increasing professional satisfaction and reducing stress, career satisfaction can be enhanced, greater worklife balance can be achieved, and long-term growth as well as achievement can be re-invigorated. 


\section{Main messages}

- Ineffective time management adversely impacts physician career satisfaction.

- Effective time management requires:

- Setting short and long-term goals

- Setting priorities among competing responsibilities

- Planning and organising activities

- Minimising exposure to circumstances that result in wasted time

- The identification and practice of time management skills will likely improve physician efficiency and career satisfaction.

\section{Current research questions}

- Does an educational programme in time management improve physician productivity?

- Does ongoing mentoring in time management improve physician productivity?

- Is time management perceived as a problem by physicians?

\section{Key references}

- Lowenstein SR. Tuesdays to write ... A guide to time management in academic emergency medicine. Acad Emerg Med 2009;16:165-7.

- Christie S. Effective time management skills for doctors. Nottingham: Developmedica, 2009.

- Jackler R. How to be organized and manage time. In: Weiss Roberts $\mathrm{L}$, ed. The academic medicine handbook-a guide to achievement and fulfillment for academic faculty. New York: Springer, 2013:17-25.

- McLauchlan C. Time management. J Accid Emerg Med 1997;14:345-6.

- Covey SR. The 7 Habits Of Highly Effective People. London: Simon \& Schuster Ltd, 1990.
Contributors CEG and SCB contributed equally to developing the idea for the manuscript, and writing and editing the manuscript. CEG performed the literature review.

Competing interests None.

Provenance and peer review Not commissioned; externally peer reviewed.

\section{REFERENCES}

1 Braddock CH III, Snyder L. The doctor will see you shortly. The ethical significance of time for the patient-physician relationship. J Gen Intern Med 2005;20:1057-62.

2 Morrison I. The future of physician's time. Ann Intern Med 2000;132:80-4.

3 Linzer M, Konrad TR, Douglas J, et al. Managed care, time pressure, and physician job satisfaction: results from the Physician Worklife Study. J Gen Intern Med 2000;15:441-50.

4 Gundersen L. Physician burnout. Ann Intern Med 2001;135:145-8.

5 Dyrbye LN, Shanafelt TD. Physician burnout: a potential threat to successful health care reform. JAMA 2011;305:2009-10.

6 Dyrbye LN, West CP, Satele D, et al. Work/home conflict and burnout among academic internal medicine physicians. Arch Intern Med 2011;171:1207-9.

7 Crosby JW. Ten time-management tips for family physicians. CMAJ 2004;170:949-50.

8 Dugdale DC, Epstein R, Pantilat SZ. Time and the patient-physician relationship. J Gen Intern Med 1999;14(Suppl 1):S34-40.

9 Jackson VP. Time management: a realistic approach. J Am Coll Radiol 2009;6:434-6.

10 Lowenstein SR. Tuesdays to write ... A guide to time management in academic emergency medicine. Acad Emerg Med 2009;16:165-7.

11 Riba MB, Riba A, Riba E. Life as a balance beam: practical ideas for balancing work and home. Acad Psychiatry 2007;31:135-7.

12 Spickard A Jr, Gabbe SG, Christensen JF. Mid-career burnout in generalist and specialist physicians. JAMA 2002;288:1447-50.

13 Shanafelt TD, Boone $S$, Tan $L$, et al. Burnout and satisfaction with work-life balance among US physicians relative to the general US population. Arch Intern Med 2012;172:1377-85

14 Christie S. Effective time management skills for doctors. Nottingham: Developmedica, 2009.

15 Jackler R. How to be organized and manage time. In: Weiss Roberts $L$, ed. The academic medicine handbook - a guide to achievement and fulfillment for academic faculty. New York: Springer, 2013:17-25.

16 McLauchlan C. Time management. J Accid Emerg Med 1997;14:345-6.

17 Covey SR, Merrill AR, Merrill RR. First things first. Free Press, 1996.

18 Brunicardi FC, Hobson FL. Time management: a review for physicians. J Nat/ Med Assoc 1996;88:581-7.

19 Covey SR. The 7 Habits Of Highly Effective People. London: Simon \& Schuster Ltd, 1990.

20 Pisano ED. Time management 101. Acad Radiol 2001;8:768-70.

21 Taylor R, ed. Academic Medicine: A Guide for Clinicians. New York: Springer, 2007:185-212.

22 Taylor R. Medical Wisdom and Doctoring: the Art of 21st Century Practice. New York: Springer, 2010.

23 Johnson SR. Personal Productivity: How to Work Effectively and Calmly in the Midst of Chaos. 2008. http://www.cvdtraining.pitt.edu/docs/Johnson2009_Essays.pdf

24 Boice R. The New Faculty Member. San Francisco: Jossey-Bass, 1992.

25 Candib LM. Making time to write? Ann Fam Med 2005:3:365-6.

26 Lemkau JP, Ahmed SM. Helping junior faculty become published scholars. Acad Med 1999:74:1264-7.

27 Cohen ML. Making the most of the daily commute. Fam Pract Manag 2007;14:56. 